\title{
Pairing Correlations around Negative- $U$ Centre in the Two-Band Model
}

\author{
A. Ciechan and K.I. Wysokiński \\ Institute of Physics and Nanotechnology Centre \\ M. Curie-Skłodowska University \\ Radziszewskiego 10, PL-20-031 Lublin, Poland
}

\begin{abstract}
The electron energy spectrum of many superconducting materials is characterised by the presence of few bands at the Fermi level. In some cases the superconducting properties seem to be dominated by single band but in others the many-band approach is essential ingredient of their description. In this paper we shall study the properties of superconducting impurity placed in a non-superconducting medium. We are interested in the evolution of superconducting correlations within non-superconducting 1- or 2-dimensional system, their dependence on the distance from impurity and changes induced by the presence of the second band and interband scattering. We use real space description of the material and Bogolyubov-de Gennes approach to superconductivity.
\end{abstract}

PACS numbers: 74.20.-z, 74.81.-g, 74.90.+n

\section{Introduction}

The orthodox, BCS theory of superconductivity developed more than half a century ago is restricted to single band systems. It has been extended to two-band situation $[1,2]$ and since then used by numerous authors to study peculiarities introduced by the second band and interactions between the bands. As one representative example we cite here the work by Kondo [3], where it has been shown that the presence of the second band with repulsive electron-electron interaction enhances the superconducting transition temperature if two bands interact with each other. Moreover, he pointed out that large interband interaction leads to vanishing of the isotope coefficient - the fact which often is used as evidence for non-phonon mechanism of superconductivity. More detailed discussion of the many-band approaches to bulk superconductors can be found in Ref. [4] and to small superconductors in [5].

It is the purpose of the present paper to extend, to the two-band case, the previous study [6] of superconducting correlations in the vicinity of single 
impurity or small grain placed in non-superconducting host. Of particular interest is the question if the two-band scenario introduces new spatial scales into the problem and how the pair correlations induced by inter-band pair scattering will depend on the distance from impurity. The existence of pair correlations does not require thermodynamic limit. They appear also in small systems, even around a single impurity with an attractive interaction (the negative- $U$ impurity) or isolated metallic grains [7] with the finite number of electrons and have recently been studied experimentally (for a review see [8]).

We assume that the system under consideration can be modelled by the discrete two-band tight-binding model in which the superconducting impurity is placed at point $\boldsymbol{r}_{0}$ :

$$
\begin{aligned}
H= & -\sum_{i j, \lambda \lambda^{\prime}, \sigma} t_{i j}^{\lambda \lambda^{\prime}} c_{i \lambda \sigma}^{+} c_{j \lambda^{\prime} \sigma}+\sum_{i, \lambda, \sigma}\left(\epsilon_{\lambda}-\mu\right) c_{i \lambda \sigma}^{+} c_{i \lambda \sigma} \\
& -\sum_{\lambda \lambda^{\prime}} U_{\lambda \lambda^{\prime}} c_{0 \lambda \uparrow}^{+} c_{0 \lambda \downarrow}^{+} c_{0 \lambda^{\prime} \downarrow} c_{0 \lambda^{\prime} \uparrow},
\end{aligned}
$$

where the subscripts $\lambda=1,2$ denote two bands, which we also call $s$ and $d$ bands, operator $c_{i \lambda \sigma}^{+}\left(c_{i \lambda \sigma}\right)$ creates (annihilates) electron with the spin $\sigma=\uparrow, \downarrow$ in site $i \equiv \boldsymbol{r}_{i}=\left(x_{i}, y_{i}\right)$ and band $\lambda . \epsilon_{\lambda}$ is the electron energy in the band $\lambda, \mu$ is the chemical potential. $t_{i j}^{\lambda \lambda^{\prime}}$ are the hopping elements and $U_{\lambda \lambda^{\prime}}>0$ attractive interactions inside a band if $\lambda=\lambda^{\prime}$ or between the bands $\lambda \neq \lambda^{\prime}$. The interband interaction we use here has the form of pair scattering.

Using mean-field Hartree-Fock-Bogolyubov decoupling and applying the Bogolyubov-Valatin transformation

$$
\begin{aligned}
& c_{i \lambda \uparrow}=\sum_{k}\left(u_{k \lambda}\left(\boldsymbol{r}_{i}\right) \gamma_{k \uparrow}-v_{k \lambda}^{*}\left(\boldsymbol{r}_{i}\right) \gamma_{k \downarrow}^{+}\right), \\
& c_{i \lambda \downarrow}=\sum_{k}\left(u_{k \lambda}\left(\boldsymbol{r}_{i}\right) \gamma_{k \downarrow}+v_{k \lambda}^{*}\left(\boldsymbol{r}_{i}\right) \gamma_{k \uparrow}^{+}\right),
\end{aligned}
$$

where $u_{k \lambda}\left(\boldsymbol{r}_{i}\right), v_{k \lambda}\left(\boldsymbol{r}_{i}\right)$ are amplitudes of electrons and holes in the band $\lambda, \gamma_{k \sigma}^{+}, \gamma_{k \sigma}$ the creation and annihilation operators of quasiparticles, we obtain the following Bogolyubov-de Gennes (BdG) equations:

$$
\begin{aligned}
& \sum_{\lambda^{\prime} j}\left[\left(\epsilon_{\lambda}-\mu\right) \delta_{\lambda \lambda^{\prime}} \delta_{i j}+V_{\lambda \lambda^{\prime}} \delta_{i j} \delta_{i 0}-t_{i j}^{\lambda \lambda^{\prime}}\right] u_{k \lambda^{\prime}}\left(\boldsymbol{r}_{j}\right) \\
& +\sum_{\lambda^{\prime}} \Delta_{\lambda^{\prime} \lambda} \delta_{i 0} v_{k \lambda}\left(\boldsymbol{r}_{i}\right)=E_{k} u_{k \lambda}\left(\boldsymbol{r}_{i}\right), \\
& -\sum_{\lambda^{\prime} j}\left[\left(\epsilon_{\lambda}-\mu\right) \delta_{\lambda \lambda^{\prime}} \delta_{i j}+V_{\lambda \lambda^{\prime}}^{*} \delta_{i j} \delta_{i 0}-t_{i j}^{\lambda \lambda^{\prime}}\right] v_{k \lambda^{\prime}}\left(\boldsymbol{r}_{j}\right) \\
& +\sum_{\lambda^{\prime}} \Delta_{\lambda^{\prime} \lambda}^{*} \delta_{i 0} u_{k \lambda}\left(\boldsymbol{r}_{i}\right)=E_{k} v_{k \lambda}\left(\boldsymbol{r}_{i}\right) .
\end{aligned}
$$

The pairing $\Delta_{\lambda \lambda^{\prime}}$ and the Hartree-Fock potentials $V_{\lambda \lambda^{\prime}}$ are in turn given in terms of the quasiparticle and quasihole amplitudes $u_{k \lambda}\left(\boldsymbol{r}_{i}\right), v_{k \lambda}\left(\boldsymbol{r}_{i}\right)$ 
as $\Delta_{\lambda \lambda^{\prime}}=-U_{\lambda \lambda^{\prime}} f_{0 \lambda}=U_{\lambda \lambda^{\prime}} \sum_{k} u_{k \lambda}\left(\boldsymbol{r}_{0}\right) v_{k \lambda}^{*}\left(\boldsymbol{r}_{0}\right)\left(1-2 f_{k}\right)$ and $V_{\lambda \lambda^{\prime}}=-U_{\lambda \lambda^{\prime}}$ $\sum_{k}\left[u_{k \lambda}^{*}\left(\boldsymbol{r}_{0}\right) u_{k \lambda^{\prime}}\left(\boldsymbol{r}_{0}\right) f_{k}+v_{k \lambda}\left(\boldsymbol{r}_{0}\right) v_{k \lambda^{\prime}}^{*}\left(\boldsymbol{r}_{0}\right)\left(1-f_{k}\right)\right]$ and $f_{k}=\left[\exp \left(\beta E_{k}\right)+1\right]^{-1}$ is the Fermi-Dirac distribution function with $\beta=1 / k_{\mathrm{B}} T$. T denotes temperature.

\section{Numerical results and discussion}

We concentrate mainly on two-dimensional systems [6] and neglect direct $s-d$ hybridisation $t_{i, i+\delta}^{\lambda \lambda^{\prime}}=t_{\lambda} \delta_{\lambda \lambda^{\prime}}$, for our main interest here is the effect of pair scattering (i.e. the effect of potential $U_{12}$ ) on the properties of the system studied. The one-dimensional system will be treated only in our discussion of the decay of correlations. We numerically solve the BdG equations (4), (5) on a linear or square lattice with periodic boundary conditions. Both the pair correlations $f_{01}, f_{02}$ and the order parameters $\Delta_{\lambda \lambda^{\prime}}$ at the impurity site appear only when states with $N$ and $N+2$ electrons are degenerate [6]. Since the parameter space of two-band model is large we shall present results for few representative sets and leave the systematic discussion to future publication.

In Fig. 1a the order parameters $\Delta_{\lambda \lambda^{\prime}}$ and mean total electron numbers in two independent $\left(U_{12}=0\right)$ bands are plotted as a function of interaction strength in first band $U_{1}=U_{11}$ (the interaction in the second band is taken to be $U_{22}=$ $\left.U_{2}=1.1 U_{1}\right)$ in two-dimensional $9 \times 9$ system. The bands are independent and the differences of the results visible in Fig. 1a for both bands can be traced back to different parameters used to describe them. It is important to realise that due to discrete spectrum of the model there exist quasi-discrete positions of chemical potential fulfilling the requirements of degeneracy of states necessary for the development of superconducting correlations. In the case of $2 d$ system and coupled bands one finds number of such states. Figure 1b shows the influence of moderate $\left(U_{12}=0.5 t\right)$ pair scattering. For the particular value of the chemical potential $\mu=-2.4 t$ both bands do show superconducting fluctuations. Let us note the existence of the threshold $|U|$ values for pairing in each of the bands. As seen in Fig. 1b coupling $U_{12}$ changes lower critical value of $|U|$ in $s$-band and induces pairing correlations between the $s$ - and $d$-bands. The number of electrons in both bands changes monotonically from 18 to 20 if we increase the interaction $U_{11}$ (with $U_{22}=1.1 U_{11}$ ). For coupled bands the dependence of $N_{1}\left(N_{2}\right)$ on $U_{1}$ is non-monotonic. This is due to pair scattering between the bands.

The order parameter being proportional to $U_{i}$ is nonzero only at the impurity site $i$. However, as mentioned earlier, the pairing correlations $f_{i \lambda}=\left\langle c_{i \lambda \downarrow} c_{i \lambda \uparrow}\right\rangle=$ $-\sum_{k} u_{k \lambda}\left(\boldsymbol{r}_{i}\right) v_{k \lambda}^{*}\left(\boldsymbol{r}_{i}\right)\left(1-2 f_{k}\right)$ take on non-zero value in the vicinity of the impurity. In Fig. 2 we show the spatial decay of the correlations around an impurity in simple 1-dimensional system consisting of single negative $U$ impurity in the middle of chain 51 atoms long. The extent of correlations does depend on the model parameters. In one-band case [6] the correlations are short ranged for $|U|$ larger than the band width. In a model with coupled bands the dependence is more complicated and the detailed study will be presented elsewhere. Figure $2 \mathrm{a}$ 

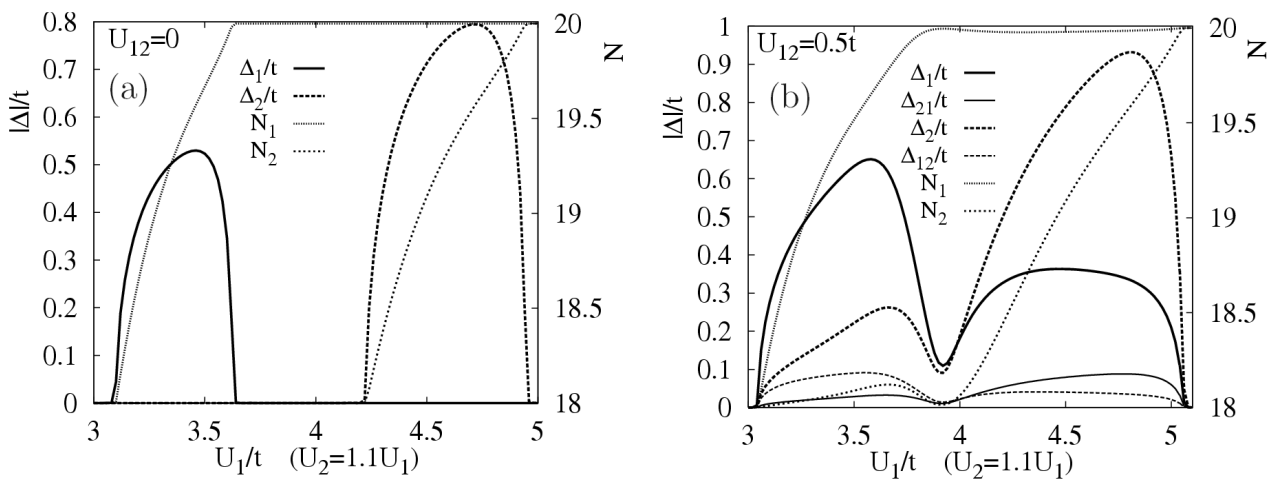

Fig. 1. (a) The order parameter $\Delta_{\lambda}$ and average number of electrons $N_{\lambda}$ in a $\lambda$ band as function of $U_{1}$ (and for $U_{2}=1.1 U_{1}$ ) for independent bands $\left(U_{12}=0\right)$. (b) The same for bands coupled by pair scattering $U_{12}=0.5 t$. The other parameters of this two-dimensional $9 \times 9$ system are $\epsilon_{1}=0, \epsilon_{2}=1.2 t, t_{1}=t, t_{2}=1.5 t$, chemical potential $\mu=-2.4 t$.

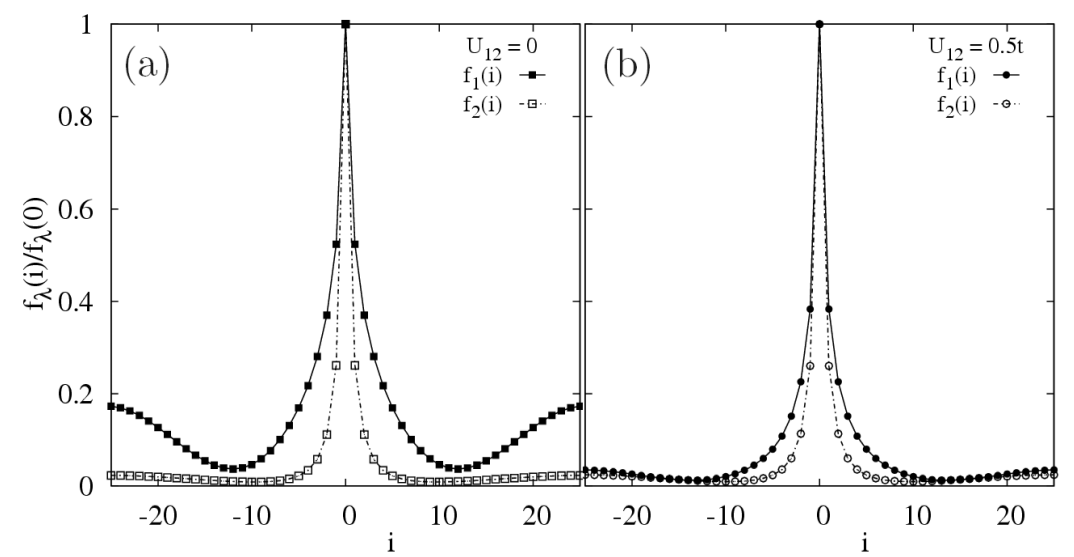

Fig. 2. Dependence of normalised superconducting correlations $f_{\lambda}(i) / f_{\lambda}(0), \lambda=1,2$ on the distance from the impurity site placed in the centre of one-dimensional ring with 51 sites. Other parameters: $\epsilon_{1}=0, \epsilon_{2}=2 t, t_{1}=t, t_{2}=2 t, U_{1}=1.5 t, U_{2}=5 t$, $\mu=-1.99 t$.

shows the dependence of normalised $f_{i \lambda}$ for two bands on the distance from the impurity. For the chosen set of parameters correlations extend over the whole system. We have found that for both interactions larger than the band widths i.e. for $U_{\lambda, \lambda}>2 z\left|t^{\lambda, \lambda}\right|$, where $z$ is the coordination number of the lattice, the correlations are short ranged and exponentially vanish with distance from the impurity. More complicated behaviour can be observed for general values of parameters. Figure $2 \mathrm{~b}$ illustrates the effect of $U_{12}$ on the correlations in the same one-dimensional system with periodic boundary conditions. The pair scattering makes the dependence in 
both bands to be similar to each other. The correlations of coupled bands extend over the whole space. Similar results are obtained for free boundary conditions.

\section{Summary and conclusions}

Our calculations indicate that the effect of the interband pair scattering shows up as:

- an increase in superconducting fluctuations (larger $\Delta_{\lambda}$ values) in the system and increase in the region of their appearance on the $\left(U_{\lambda}, \mu\right)$ plane,

- new contributions $\left(\Delta_{21}\right.$ and $\left.\Delta_{12}\right)$ to order parameters $\Delta_{1}$ and $\Delta_{2}$ depend on the interaction $U_{12}$ and the density of states in the other band,

- due to proximity effect the pairing correlations may extend over the whole system. The interband scattering smoothes out their spatial structure in two bands.

It would be interesting to test some of these predictions by measuring the local gaps of one-band and (strongly) two-band impurities or grains (like $\mathrm{MgB}_{2}$ ) placed in a non-superconducting host.

\section{Acknowledgments}

This work was partially supported by the grant no. N N202 187833 .

\section{References}

[1] H. Suhl, B.T. Matthias, L.R. Walker, Phys. Rev. Lett. 3, 552 (1959).

[2] M. Suffczynski, Phys. Rev. 128, 1538 (1962).

[3] J. Kondo, Prog. Theor. Phys. 29, 1 (1963).

[4] N. Bock, D. Coffey, Phys. Rev. B 76, 174513 (2007).

[5] A. Bianconi, J. Supercond. 18, 637 (2005).

[6] Y. Dubi, Phys. Rev. B 75, 094510 (2007).

[7] A. Ciechan, K.I. Wysokiński, Acta Phys. Pol. A 111, 467 (2007).

[8] J. von Delft, D.C. Ralph, Phys. Rep. 345, 4087 (1996). 